\title{
ESTUDIO COMPARATIVO DEL TRATAMIENTO ORTÉSICO EN LAS FRACTURAS TORACO-LUMBOSACRAS SEGÚN LA GRAVEDAD DEL TRAUMA
}

\author{
ESTUDO COMPARATIVO DO TRATAMENTO ORTÓTICO NAS FRATURAS \\ TORACO-LOMBOSACRO SEGUNDO A GRAVIDADE DO TRAUMA
}

COMPARATIVE STUDY ON ORTHOTIC TREATMENT OF THORACO-LUMBO-SACRAL FRACTURES ACCORDING TO SEVERITY OF TRAUMA

Patricio Manzone ${ }^{1,2}$, Julio Stefanizzi ${ }^{3}$, Eduardo Mariño Ávalos ${ }^{4}$, Silvia Manzone Barranco ${ }^{2}$, Claudia Ihlenfeld ${ }^{2}$

\section{RESUMEN}

Objetivo: Determinar si la gravedad del trauma en lesiones toracolumbosacras mayores estables permite decidir la selección del tipo de ortesis en un tratamiento ortopédico. Métodos: Estudio Retrospectivo de casos 12/1990 - 12/2006 (16 años). Criterios de Selección: 1) Seguimiento mínimo: 2 años. 2) Estudios radiológicos convencionales completos. 3) Ausencia de Litigio. 4) Tratamiento ortésico con TLSO a medida para los traumas de alta energía cinética y con ortesis prefabricadas para los de baja energía. 5) Tratamiento efectuado o supervisado por el autor Sénior. Evaluación por observadores independientes de Parámetros Geométricos (ángulo de Cobb sagital, cifosis vertebral, grado de colapso vertebral) pretratamiento y seguimiento en Rx simple, y Parámetros Funcionales (Dolor según SRS, Índice de Oswestry, Retorno a la Actividad Previa). Subdivisión de los diferentes tipos de fracturas (según AO y Denis) en Alta (Grupo A) y Baja Energía [Grupo B] de acuerdo con la energía cinética del trauma. Comparación de Parámetros Geométricos con Grupo Control. Análisis Estadístico: chi cuadrado y t-test de Student. Resultados: 41 pacientes (44 fracturas] tratados (23 mujeres/18 varones), con 25 fracturas Grupo "A", y 19 Grupo "B". Edad promedio: 46 años (12 - 83). Seguimiento promedio: 4,5 años (2.2 - 15.5). Localización predominante: T11 - L2. Tipos Predominantes: tipo A (AO) o por compresión y por estallido. No hubo diferencias significativas en las mediciones efectuadas en cada grupo pretratamiento y al seguimiento. La única diferencia significativa entre grupos fue en la cifosis vertebral inicial tanto en general como según la clasificación $\mathrm{AO}$ entre los tipos A de alta y baja energía. La comparación al seguimiento de los parámetros geométricos entre grupo control y grupos A y B así como entre grupo control y cada tipo (AO/Denis) subdivididos en alta o baja energía, arrojó siempre diferencias significativas. Los parámetros funcionales al seguimiento mostraron siempre puntuaciones promedio buenas, con variaciones significativas entre grupos A y B. El retorno a la actividad previa fue del 90,6\%, sin diferencias entre trabajadores de esfuerzo físico y de escritorio. Conclusiones: Es posible lograr un Resultado Clínico Funcional satisfactorio a mediano plazo en las lesiones toracolumbosacras mayores estables seleccionando el tipo de ortesis según que el trauma sea de alta o baja energía cinética. Los resultados clínicos funcionales parecen ser mejores en los casos de Trauma de Alta Energía. Sin embargo, este tratamiento no mejora ni empeora los parámetros radiológicos sagitales.

Descriptores: Fracturas de la columna vertebral; Vértebras torácicas; Aparatos ortopédicos; Estudios retrospectivos.

\section{RESUMO}

Objetivo: Determinar se a gravidade do trauma toraco-lombo-sacro estável permite decidir o tipo de órteses na seleção do tratamento ortopédico. Métodos: Estudo retrospectivo (16 anos). Critérios de inclusão: 1) Seguimento mínimo: 2 anos. 2) Estudos radiológicos convencionais completos. 3) Ausência de litígio. 4) Tratamento com TLSO feito à medida para trauma de alta energia cinética e órteses pré-fabricadas para trauma de baixa energia. 5) Tratamentos realizados ou supervisionados pelo autor sênior. Avaliação por observadores independentes de parâmetros geométricos (Cobb sagital, cifose da vértebra, grau de colapso vertebral anterior) iniciais e ao seguimento, e parâmetros funcionais (dor de acordo com a SRS, índice de Oswestry, retorno à atividade anterior). Subdivisão de diferentes tipos de fraturas (AO e Denis) proporcional à energia cinética do trauma, em (grupo A) alta energia e (grupo B) baixa energia. Comparação com o grupo de controle. Análise estatística: qui quadrado e teste tStudent. Resultados: Estudos até 41 pacientes (44 fraturas, 23 mulheres/18 homens), 25 fraturas grupo "A" e 19 grupo "B". Média de idade: 46 anos (12-83). Seguimento médio: 4,5 anos (2,2 -15,5). Localização predominante: T11 - L2. Tipos predominantes: Tipo A (AO) ou por compressão e estalido. Não houve nenhuma diferença significativa entre medições iniciais e seguimento. A única diferença significativa entre os grupos foi a cifose inicial da coluna vertebral. Sempre houve dife-

\footnotetext{
1. Unidad de Patologia Espinal - Servicio de Ortopedia y Traumatologia - Hospital “Dr. Avelino Castelán” - Juan B. Justo 1150 - (3500) Resistencia - Argentina.

2. Centro Nicolás Andry - Monteagudo 207 - (3500) Resistencia - Argentina.

3. Servicio de Ortopedia \&Traumatología - Hospital "Dr. Julio C. Perrando"- Av. 9 de Julio 1050 - (3500) Resistencia - Argentina.

4. Servicio de Ortopedia y Traumatologia - Hospital Central de Formosa - (3600) Formosa - Argentina.

Trabalho realizado no Centro Nicolás Andry - Monteagudo 207 - (3500) Resistencia - Argentina e Hospital Dr. Julio C. Perrando - (3500) Resistencia - Argentina

Correspondência: Hospital Dr. Avelino Castelán - Centro Nicolás Andry - Monteagudo 207 - (3500) Resistencia - Argentina - E-mail: manzonepatricio@ hotmail.com
} 
renças na comparação dos parâmetros geométricos do grupo controle e os grupos $\mathrm{A}$ e B, e entre o grupo controle e cada tipo (AO/Denis) subdividido em alta ou baixa energia. As pontuações dos parâmetros funcionais finais sempre foram boas, com variações significativas entre os grupos A e B. Conclusões: É possível um bom resultado funcional em lesões toraco-lombo-sacrais estáveis, selecionando o tipo de órteses de acordo com a energia cinética do trauma. Esses resultados parecem ser melhores em traumas de alta energia tratados com dispositivos ortopédicos feitos à medida. No entanto, o tratamento ortésico diferenciado de acordo com a energia do trauma não altera os parâmetros radiológicos sagitais.

Descriptores: Fraturas da coluna vertebral; Vértebras torácicas; Aparelhos ortopédicos; Estudos retrospectivos.

\section{ABSTRACT}

Objective: To determine whether the severity of stable thoraco-lumbo-sacral trauma is useful for deciding the selection of brace type in orthopedic treatment. Methods: Retrospective study (16 years length). Inclusion criteria: 1) Minimum follow-up: 2 years. 2) Complete conventional radiologic studies. 3) No litigation. 4) TLSO custom-made treatment for high kinetic energy trauma and pre-fabricated orthoses for low energy one. 5) Treatment performed or supervised by the senior author. Evaluation by independent observers of Geometric Parameters (sagittal Cobb, vertebral kyphosis, anterior vertebral collapse) initially and at follow-up, and Functional Parameters (SRS pain scale, Oswestry Index, Return to Previous Activity). Subdivision of different fractures types (of AO and Denis classifications) in High (Group A) and Low Energy (Group B) according to the amount of kinetic energy of trauma. Comparison with a control group. Statistical analysis: chi square and Student t-test. Results: Forty-one patients were studied (44 fractures, 23 females/18 males), 25 fractures Group A and 19 Group B. Average age: 46 years (12 - 83). Average follow-up: 4.5 years (2.2 - 15.5). Predominant location: $T 11$ - L2. Predominant types: Type A (AO) or compression and burst. There were no significant differences between initial and follow-up measurements. The only significant difference between groups was in the initial vertebral kyphosis. However, there were always differences when comparing the Geometric Parameters between control group and groups $A$ and $B$, and between control group and each type (AO/Denis) subdivided into high or low energy. The final functional parameters scores were always good, with significant variations between groups $A$ and $B$. Conclusions: A satisfactory functional result in stable thoraco-lumbo-sacral injuries is possible by selecting the type of brace according to the kinetic energy involved. Results appear to be better in High Energy Trauma treated with custom-made orthosis. However, the orthotic treatment according to trauma energy does not change the sagittal radiographic parameters.

Keywords: Spinal fractures; Thoracic vertebrae; Orthotic devices; Retrospective studies.

\section{INTRODUCCIÓN}

El tratamiento de las fracturas evolucionó hacia una modalidad más quirúrgica en los últimos 50 años, a despecho de las buenas posibilidades de curación biológica con métodos conservadores¹. Las Fracturas tóraco-lumbares no escaparon a esta tendencia incrementándose mucho el número de las que se operan. Sin embargo, y aunque todavía persista cierta controversia ${ }^{2-4}$, el tratamiento incruento fue el "gold standard" por muchos años. Este se indica actualmente en lesiones menores y aquellas mayores consideradas estables, sin potencial para deformidad progresiva o lesión nerviosa ${ }^{5}$, y es habitualmente aceptado que las mismas pueden ser tratadas con ortesis ${ }^{6}$.

Ahora bien, en el trauma de raquis - como en otros traumas - el tipo y la magnitud de la lesión son determinados por la cantidad de movimiento y la energía cinética asociadas con el impacto, las características de los tejidos subyacentes y el ángulo de solicitación mecánica aplicado ${ }^{7-10}$. Por ende, un mismo patrón de lesión anátomo-radiológica según las clasificaciones existentes puede ser diferente según que el trauma sea de alta energía cinética (ejemplo, accidente de vía pública en hueso normal) o de baja energía (ejemplo, caída sentado de un individuo con hueso insuficiente), puesto que sus lesiones asociadas pueden ser diferentes, y la cantidad de energía y las fuerzas deformantes que se transmitieron al raquis lo fueron. No sería lógico usar el mismo tipo de ortesis en ambos casos, aún si radiológicamente ambas lesiones son comprendidas en el mismo grupo de la clasificación. Aquellas de más alta energía requerirían en teoría ortesis que logren una mayor contención y, eventualmente, corrección; a diferencia de las de baja energía cuyos requerimientos en dicho sentido serían menores. Así, las TLSO hechas a partir de moldes personalizados tomados en mesas de columna (que son las que exhiben mejor control de las fuerzas deformantes y mayor contención e inmovilización global ${ }^{11}$ deberían al menos hipotéticamente usarse en los traumas más severos (de alta energía), mientras que aquellas prefabricadas de menores ventajas biomecánicas teóricas ${ }^{12}$ deberían reservarse para los traumas menos severos (de baja energía).

Nuestro objetivo en este trabajo fue entonces evaluar si la severidad del trauma en las lesiones tóraco-lumbosacras mayores estables puede influir en la selección del tipo de ortesis para el tratamiento no quirúrgico, lográndose o no resultados satisfactorios a mediano plazo con distintos esquemas terapéuticos.

\section{MÉTODOS}

Este es un estudio retrospectivo, con revisión de historias clínicas y estudios de imágenes de pacientes con Fracturas TóracoLumbosacras tratadas de manera incruenta del 12/1990 - 12/2006 (16 años).

Los Criterios de Inclusión para el presente trabajo fueron: 1) Dos (2) años de seguimiento mínimo. 2) Estudios radiológicos convencionales completos (pre y postratamiento). 3) Ausencia de Litigio labora ${ }^{5,13,14}$.4) Seguimiento clínico alejado. 5) Tratamiento efectuado o supervisado por el autor Senior (PM).

La serie se dividió en dos subgrupos acorde a si el trauma fue de Alta Energía Cinética (Grupo "A") o de Baja Energía Cinética 
(Grupo "B") ${ }^{7}$. Se utilizó el factor etiológico como criterio de división: los accidentes involucrando automotores donde la velocidad del vehículo colisionante fue superior a $47.4 \mathrm{~km} / \mathrm{h}^{15}$, los impactos directos con elementos de alta masa, los traumas en deportes con alta velocidad y las caídas de altura significativa $\left(>\right.$ de $6 \mathrm{~m}^{16}$ se incluyeron en el grupo de Alta Energía; mientras que en el de Baja Energía se incluyeron las caídas de menos de $2 \mathrm{~m}$ de altura y los traumas directos por elementos de baja masa ${ }^{7}$.

El tratamiento incluyó siempre reposo en cama breve seguido por ortesis rígida a posteriori que varió según las características de las fracturas: para las de Alta Energía fue una TLSO termoplástica hecha a medida (cuyo molde se tomó al momento de la confección del corset de yeso temporario ${ }^{17,18}$ utilizado hasta la provisión del corset), mientras que para las de Baja Energía fue una Ortesis en Hiperextensión prefabricada (Corset tipo Jewett). La ambulación bajo protección fue autorizada a la provisión de la ortesis en ambos casos $^{5,17,19-22}$.

Se usaron para el análisis inicial de todos los casos el score de White y Panjabi ${ }^{23}$, el índice de Farcy ${ }^{24}$, el score del TLISS ${ }^{25}$, y las clasificaciones de Denis ${ }^{26,27}$ y la de la $\mathrm{AO}^{28}$. Para las lesiones sacras se utilizaron las clasificaciones de Denis ${ }^{26}$ y de Schmidek ${ }^{29}$. Se utilizaron las mediciones iniciales sagitales más aceptadas y de escasa variabilidad intra e interobservador ${ }^{30-33}$ : ángulo de Cobb, Roy Camille y Weinstein ${ }^{34-36}$, y grado colapso ("ABC" = anterior body compression) ${ }^{37}$.

Se utilizó un Grupo Control (Grupo "C") radiológico para los valores angulares sagitales promedio de nuestra población: una serie de 19 adultos (10 mujeres/9 varones) sin síntomas ni patologías raquídeas ostensibles, ni déficit neurológico de ningún tipo. Todas las mediciones las realizaron observadores independientes.

El resultado clínico funcional se evaluó según el Dolor secuelar al seguimiento con la Escala de la Scoliosis Research Society ${ }^{38}$ y el Índice de Discapacidad de Oswestry Validado para la Argentina ${ }^{39}$, así como en el Retorno a la Actividad Previa al Trauma.

El análisis estadístico se realizó con el chi cuadrado para las variables cualitativas y el t-test de Student para las cuantitativas ${ }^{40-42}$, utilizando el programa EPI INFO 6 y seleccionando el valor 0.05 como valor de significación estadística ${ }^{41-43}$

\section{RESULTADOS}

Durante el período señalado fueron tratados de manera ortopédica 64 pacientes por Fracturas Tóraco-lumbosacras, cumpliendo con los criterios de selección 41 pacientes (44 fracturas) que constituyen la presente serie. Los 41 casos se agrupan en 24 pacientes (25 fracturas) con Trauma de Alta Energía (Grupo "A"), y 17 pacientes (19 fracturas) con Traumas de Baja Energía (Grupo "B).

El promedio de edad inicial de la serie fue de 46 años (12 - 83), con una diferencia estadísticamente significativa ( $p$ 0.0001) entre las edades promedios de los dos grupos: Grupo A, 36 años (12 - 62) y Grupo B, 61.5 años (22 - 83). La distribución por sexos arrojó 23 mujeres/18 varones en la serie global, otra vez con una diferencia significativa ( $p$ 0.025) entre ambos subgrupos: más varones en el grupo A y más mujeres en el $\mathrm{B}$.

El promedio de seguimiento general de la serie fue de 4.5 años (2.24 a 15.5), no existiendo diferencias significativas entre ambos grupos (A y B); tampoco las hubo cuando se compararon los diferentes tipos de las clasificaciones AO y de Denis discriminados cada uno en Alta y Baja energía (Tabla 4).

La localización de las fracturas fue predominantemente entre T11 y L2 (Figura 1). En el Figura 2 pueden verse las Actividades desempeñadas previas al trauma. La etiología estuvo marcada por los accidentes de la vía pública (19 casos) y las caídas (17 casos) (Figura 3). Predominaron las fracturas por compresión o flexión y las fracturas por estallido. Solo hubo 3 pacientes con déficit neurológico: 2 con síndromes radiculares y 1 con signos esfinterianos.

En ninguno de los valores comparados entre pre-tratamiento y seguimiento hubo diferencias estadísticamente significativa (Tabla 1). Cuando el análisis comparativo se hizo para cada tipo de fractura según las clasificaciones AO y Denis discriminadas en Alta o Baja Energía, tampoco hubo diferencias entre pre-tratamiento y seguimiento con la excepción del valor de la cifosis vertebral para las fracturas tipo A de Magerl de Alta Energía, en donde la cifosis

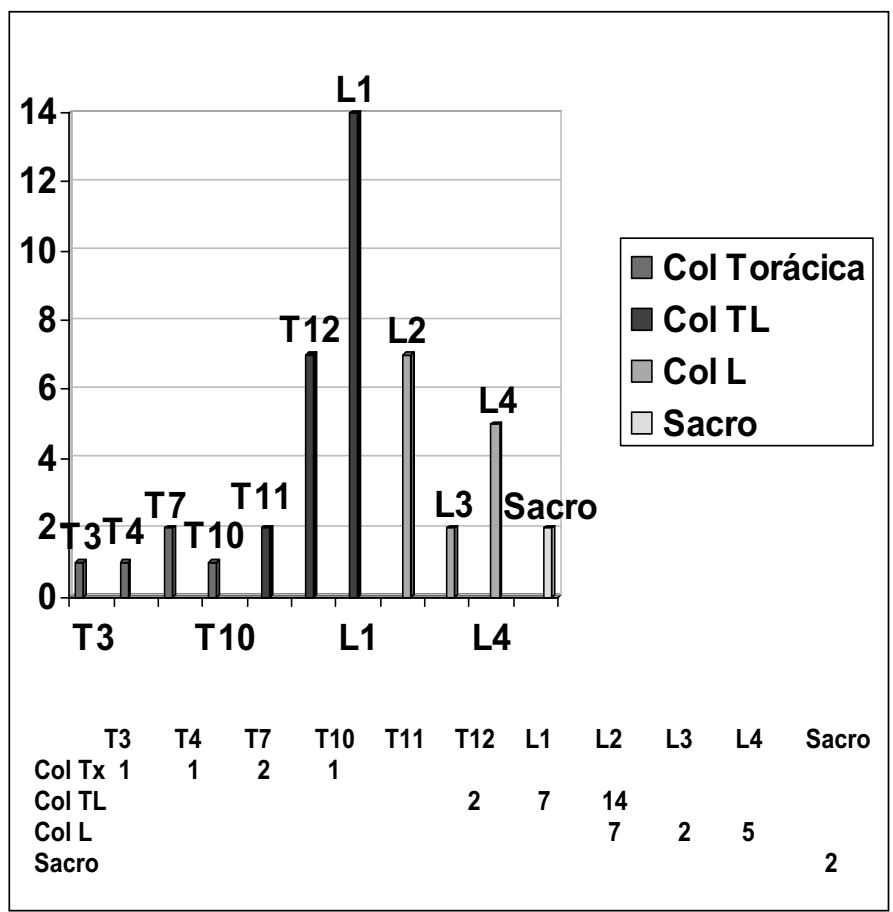

Figura 1. Localización de las fracturas.

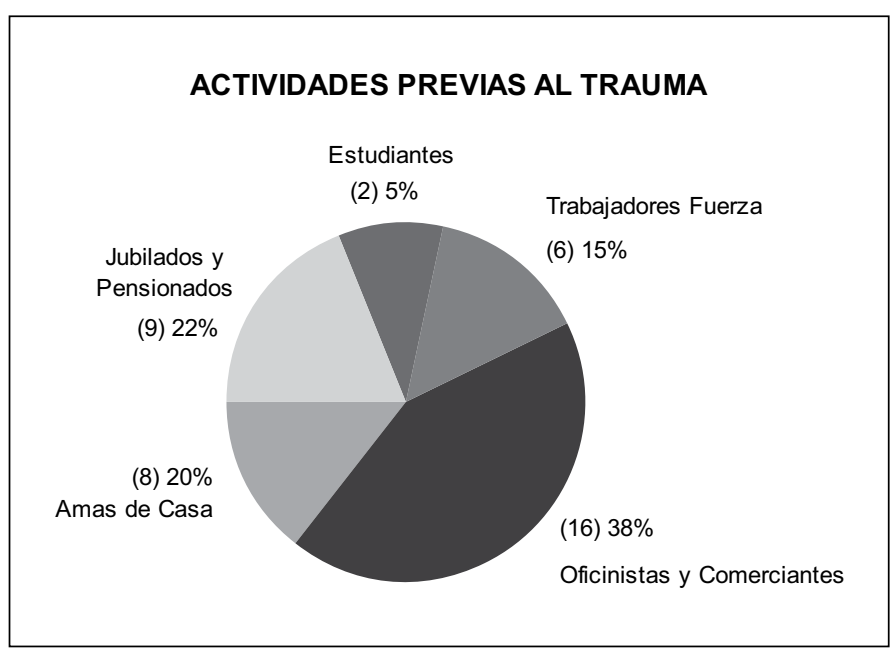

Figura 2. Actividades previas al trauma. 


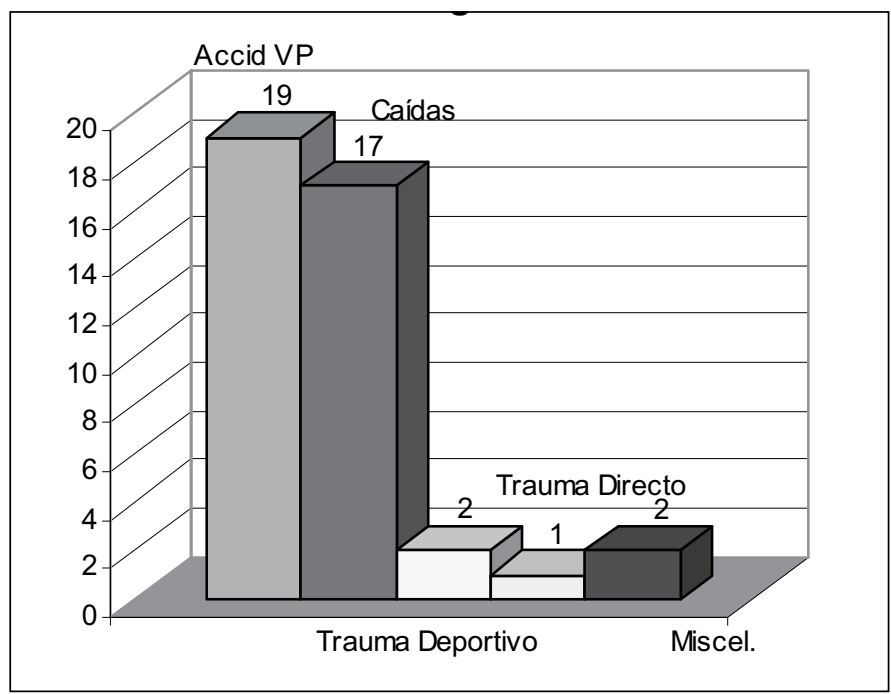

Figura 3. Etiologías

Tabla 1. Comparación de mediciones radiológicas pre-tratamiento y al seguimiento.

\begin{tabular}{|c|c|c|c|c|}
\hline & $\begin{array}{c}\text { PreTratamiento } \\
\text { [Rango] }\end{array}$ & $\begin{array}{l}\text { Seguimiento } \\
\text { [Rango] }\end{array}$ & $\begin{array}{c}\text { Valor } p \\
\text { [paired } \\
t \text { test] }\end{array}$ & $\begin{array}{c}\text { Significancia } \\
\text { Estadística }\end{array}$ \\
\hline Cifosis Vertebral & $16.4^{\circ}\left[3^{\circ}-37^{\circ}\right]$ & $\begin{array}{c}14.9^{\circ} \\
{\left[3^{\circ}-32^{\circ}\right]}\end{array}$ & 0.2116 & No \\
\hline $\begin{array}{c}\text { Cifosis } \\
\text { Segmentaria }\end{array}$ & $11^{\circ}\left[3^{\circ}-22^{\circ}\right]$ & $\begin{array}{c}11.1^{\circ} \\
{\left[2^{\circ}-25^{\circ}\right]}\end{array}$ & 0.9089 & No \\
\hline $\begin{array}{l}\text { Cifosis Regional } \\
\text { según Roy } \\
\text { Camille }\end{array}$ & $13.2^{\circ}\left[3^{\circ}-35^{\circ}\right]$ & $\begin{array}{c}14.4^{\circ} \\
{\left[1^{\circ}-35^{\circ}\right]}\end{array}$ & 0.6216 & No \\
\hline $\begin{array}{c}\text { Ángulo de Cobb } \\
\text { de la Cifosis }\end{array}$ & $17.2^{\circ}\left[2^{\circ}-35^{\circ}\right]$ & $\begin{array}{c}17.3^{\circ} \\
{\left[2^{\circ}-33^{\circ}\right]}\end{array}$ & 0.9072 & No \\
\hline $\begin{array}{c}\text { Porcentaje de } \\
\text { Compresión } \\
\text { Anterior del } \\
\text { Cuerpo Vertebral } \\
\text { ["ABC"] }\end{array}$ & $\begin{array}{c}0.25 \\
{[0.01-0.86]}\end{array}$ & $\begin{array}{c}0.24 \\
{[0.03-0.8]}\end{array}$ & 0.8246 & No \\
\hline Índice de Farcy & $19.5^{\circ}\left[2^{\circ}-38^{\circ}\right]$ & No Medido & - & - \\
\hline $\begin{array}{c}\text { Score de } \\
\text { Inestabilidad de } \\
\text { White \& Panjabi }\end{array}$ & $3.2[2-7]$ & No Medido & - & - \\
\hline Score de TLISS & $2.3[0-8]$ & No Medido & - & - \\
\hline
\end{tabular}

vertebral mejoró en promedio de $18.9^{\circ}$ inicialmente a $14.4^{\circ}$ al seguimiento ( $p$ 0.037).

Los dos Grupos A y B presentaron valores similares y comparables en las mediciones pre-tratamiento y al seguimiento. La única diferencia significativa que se encontró es en el ángulo de cifosis vertebral inicial (Tablas 2 y 3). Cuando dicha diferencia se

Tabla 3. Comparación entre Grupos de Alta (Grupo A) \& Baja (Grupo B) Energía: Mediciones al Seguimiento.

\begin{tabular}{c|c|c|c|c}
\hline & Grupo A & Grupo B & $\begin{array}{c}\text { Valor } \mathbf{p} \\
\text { [unpaired t } \\
\text { test] }\end{array}$ & $\begin{array}{c}\text { Significación } \\
\text { Estadística }\end{array}$ \\
\hline Cifosis Vertebral & $14.2^{\circ}$ & $15.9^{\circ}$ & 0.5025 & NO \\
\hline $\begin{array}{c}\text { Cifosis } \\
\text { Segmentaria }\end{array}$ & $11.1^{\circ}$ & $11.1^{\circ}$ & 0.9789 & NO \\
\hline $\begin{array}{c}\text { Cifosis Regional } \\
\text { Según Roy Camille }\end{array}$ & $14.3^{\circ}$ & $14.5^{\circ}$ & 0.9531 & NO \\
\hline $\begin{array}{c}\text { Ángulo De Cifosis } \\
\text { Según Cobb }\end{array}$ & $15.5^{\circ}$ & $20.4^{\circ}$ & 0.1166 & NO \\
\hline $\begin{array}{c}\text { Porcentaje De } \\
\text { Compresión } \\
\text { Anterior Del } \\
\text { Cuerpo Vertebral }\end{array}$ & 0.30 & 0.41 & 0.1175 & NO \\
\hline
\end{tabular}

analizó para los tipos de fracturas según la clasificaciones y según la severidad del trauma, la diferencia fue estadísticamente significativa en los valores pre-tratamiento solamente entre los tipo A de Magerl de Alta y de Baja energía (promedios de $18.9^{\circ}$ y $13.38^{\circ}$ respectivamente; $p=0.04$ ).

Cuando se compararon los resultados radiológicos al seguimiento para cada tipo de fractura entre casos de Alta Energía y de Baja Energía, tampoco se hallaron diferencias estadísticamente significativas.

La comparación al seguimiento de las mediciones de la cifosis vertebral, el ángulo de Cobb y el ABC entre grupo control y traumas de alta y baja energía, arrojó siempre diferencias significativas tanto para la serie global como cuando el análisis se hizo discriminado por tipos de fracturas según ambas clasificaciones (AO y Denis) (Tabla 4).

En cuanto a los resultados clínicos, el resultado funcional basado en el dolor secuelar y en el Índice de Oswestry ${ }^{38,39}$ arrojó puntuaciones promedio buenas, con variaciones estadísticamente

Tabla 2. Comparación entre Grupos de Alta (Grupo A) \& Baja Energía (Grupo B): Mediciones Pretratamiento.

\begin{tabular}{c|c|c|c|c}
\hline & Grupo A & Grupo B & Valor p [unpaired t test] & Significación Estadística \\
\hline Cifosis Vertebral & $17.8^{\circ}$ & $13.5^{\circ}$ & 0.0413 & $\mathrm{SI}$ \\
\hline Cifosis Segmentaria & $11^{\circ}$ & $11.1^{\circ}$ & 0.9360 & $\mathrm{NO}$ \\
\hline Cifosis Regional Según Roy Camille & $14.4^{\circ}$ & $10.8^{\circ}$ & 0.1188 & $\mathrm{NO}$ \\
\hline Ángulo De Cifosis Según Cobb & $16^{\circ}$ & $19.3^{\circ}$ & 0.1963 & $\mathrm{NO}$ \\
\hline $\begin{array}{c}\text { Porcentaje De Compresión Anterior Del } \\
\text { Cuerpo Vertebral }\end{array}$ & 0.33 & 0.32 & 0.8748 & $\mathrm{NO}$ \\
\hline Score de White \& Panjabi & 3.4 & 2.9 & 0.3294 & $\mathrm{NO}$ \\
\hline Score de TLISS & 2.6 & 1.6 & 0.0905 & $\mathrm{NO}$ \\
\hline Índice De Farcy & 20.5 & 17.9 & 0.2962 & $\mathrm{NO}$ \\
\hline
\end{tabular}


Tabla 4. Comparación de Mediciones al Seguimiento entre Población Control [Grupo C] y Distintos Tipos de Fracturas según las Clasificaciones AO y de Denis, discriminados según la Energía del Trauma.

\begin{tabular}{c|c|c|c}
\hline & $\begin{array}{c}\text { Cifosis } \\
\text { Vertebral }\end{array}$ & $\begin{array}{c}\text { Ángulo de } \\
\text { Cifosis según } \\
\text { Cobb }\end{array}$ & ABC \\
\hline Grupo Control & $3.7^{\circ}\left(1^{\circ}-9^{\circ}\right)$ & $7.6^{\circ}\left(2^{\circ}-14^{\circ}\right)$ & $\begin{array}{c}0.15 \\
(-0.01 \mathrm{a}+0.96)\end{array}$ \\
\hline $\begin{array}{c}\text { Tipo A [AO] Alta } \\
\text { Energia }\end{array}$ & $\begin{array}{c}14.45^{\circ} \\
\left(5^{\circ}-32^{\circ}\right)\end{array}$ & $14.85^{\circ}\left(3^{\circ}-33^{\circ}\right)$ & $0.30(0.06-0.74)$ \\
\hline $\begin{array}{c}\text { Tipo A [AO] Baja } \\
\text { Energia }\end{array}$ & $\begin{array}{c}15.54^{\circ} \\
\left(3^{\circ}-31^{\circ}\right)\end{array}$ & $20.36^{\circ}\left(2^{\circ}-31^{\circ}\right)$ & $0.40(0.03-0.8)$ \\
\hline $\begin{array}{c}\text { Fract. Tipo } \\
\text { Compresión [Denis] } \\
\text { Alta Energía }\end{array}$ & $\begin{array}{c}13.5^{\circ}\left(5^{\circ}-26^{\circ}\right) \\
15.9^{\circ}\left(5^{\circ}-33^{\circ}\right)\end{array}$ & $0.25(0.07-0.62)$ \\
\hline $\begin{array}{c}\text { Fract. Tipo } \\
\text { Compresión [Denis] } \\
\text { Baja Energía }\end{array}$ & $\begin{array}{c}15.08^{\circ} \\
\left(4^{\circ}-26^{\circ}\right)\end{array}$ & $20.5^{\circ}\left(2^{\circ}-31^{\circ}\right)$ & $0.39(0.03-0.80)$ \\
\hline $\begin{array}{c}\text { Burst [Denis] Alta } \\
\text { Energía }\end{array}$ & $\begin{array}{c}16.7^{\circ} \\
\left(10^{\circ}-32^{\circ}\right)\end{array}$ & $12.5^{\circ}\left(3^{\circ}-23^{\circ}\right)$ & $0.41(0.22-0.74)$ \\
\hline $\begin{array}{c}\text { Burst [Denis] Baja } \\
\text { Energía }\end{array}$ & $\begin{array}{c}20.5^{\circ} \\
\left(10^{\circ}-31^{\circ}\right)\end{array}$ & $24.5^{\circ}\left(22^{\circ}-27^{\circ}\right)$ & $0.48(0.28-0.68)$ \\
\hline
\end{tabular}

significativas entre los grupos de A y B cuando los mismos se compararon globalmente, mostrando los pacientes de este último grupo resultados menos favorables (Tabla 5). Sin embargo, cuando la comparación se hizo por tipo de fractura de ambas clasificaciones no hubo diferencias estadísticas marcadas en los resultados en cada tipo entre los de Alta y Baja energía.

Tabla 5. Resultados Clínicos según el Dolor Secuelar y el Oswestry Disbility Index validado para Argentina.

\begin{tabular}{c|c|c|c|c|c}
\hline & $\begin{array}{c}\text { Promedio } \\
\text { Puntuación } \\
\text { Global de } \\
\text { la Serie } \\
\text { (rango) }\end{array}$ & $\begin{array}{c}\text { Promedio } \\
\text { Puntuación } \\
\text { de Grupo A } \\
\text { (rango) }\end{array}$ & $\begin{array}{c}\text { Promedio } \\
\text { Puntuación } \\
\text { de Grupo } \\
\text { B (rango) }\end{array}$ & $\begin{array}{c}\text { Valor } \mathbf{p} \\
\text { (unpaired } \\
\mathbf{t} \text { test) }\end{array}$ & $\begin{array}{c}\text { Diferencia } \\
\text { Estadísti- } \\
\text { camente } \\
\text { Significa- } \\
\text { tiva }\end{array}$ \\
\hline $\begin{array}{c}\text { Score de } \\
\text { SRS }\end{array}$ & $4.4(2$ a 5$)$ & $4.7(3$ a 5$)$ & $3.9(2$ a 5$)$ & 0.005 & SI \\
\hline $\begin{array}{c}\text { Oswestry } \\
\text { Disabil- } \\
\text { ity Index } \\
\begin{array}{c}\text { Validación } \\
\text { Argentina }\end{array}\end{array}$ & $\begin{array}{c}15 \\
(10 ~ 40)\end{array}$ & $\begin{array}{c}11.4 \\
(10 \text { a } 24)\end{array}$ & $\begin{array}{c}20.2 \\
(10 \text { a } 40)\end{array}$ & 0.0002 & SI \\
\hline
\end{tabular}

En cuanto al retorno a la actividad previa al trauma, excluyendo los jubilados y pensionados fue del 90.6\% en la serie global (Figura 4). Si bien el número en cada grupo no fue muy importante, aparentemente no existió diferencia estadísticamente significativa entre los trabajadores de esfuerzo y de escritorio con el test exacto de Fisher (p 0.08). Tampoco hubo diferencias estadísticamente significativas cuando el retorno a la actividad previa entre trabajadores de esfuerzo y de escritorio se analizó con el mismo test en los diferentes grupos: grupo A ( $p$ 0.14) y grupo B (p 1.00).

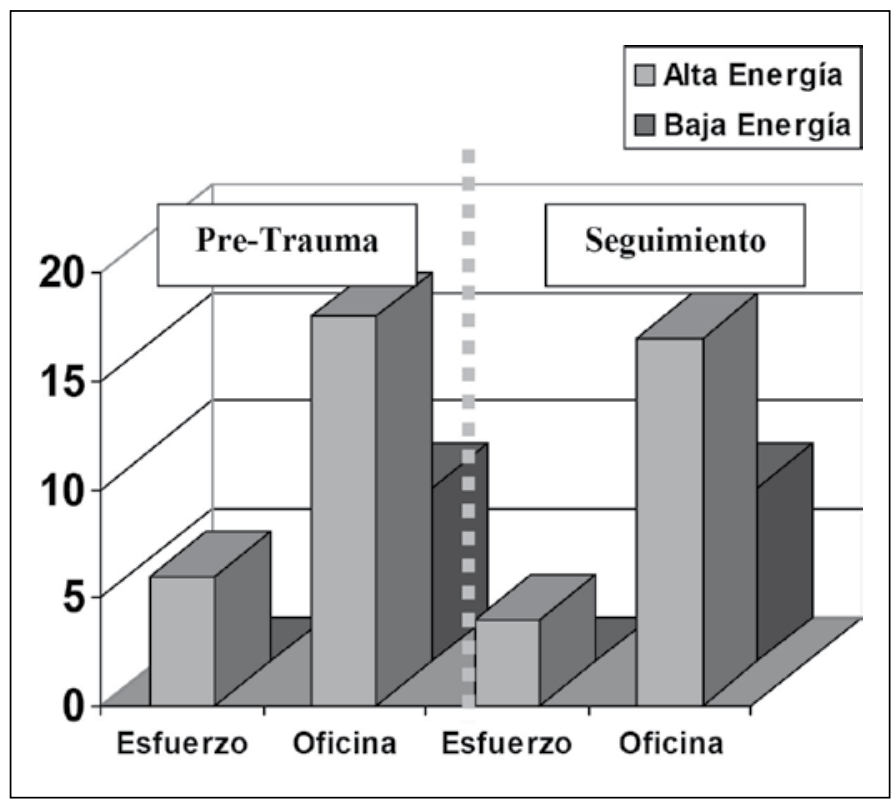

Figura 4. Retorno Laboral.

\section{DISCUSIÓN}

Ninguna clasificación de las fracturas vertebrales tóraco-lumbares demostró hasta ahora abarcar completamente el universo de las fracturas del raquis ${ }^{44,46}$; las más frecuentemente usadas son las de Denis, la de MacAfee, la de Gaines, la de Magerl y el TLISS 28,47-51. Pero si bien la biomecánica de la transmisión de fuerzas puede ser fácil y rápidamente demostrada individualmente para los diferentes componentes de la columna, un modelo universalmente aceptado que demuestre los patrones de lesiones en una columna intacta todavía no existe ${ }^{7}$.

Desde el concepto de inestabilidades óseas y ligamentarias ${ }^{52}$, creemos válido el tratamiento incruento en lesiones estables o con inestabilidad exclusivamente ósea, sin déficit neurológico y sin factor de compresión actual.

Así, los resultados reportados para Fractura Estallidos Estables son excelentes y se comparan favorablemente con los del Tratamiento Quirúrgico 2,4-6,14,17,19-21,27,36,53-64; aún con lesión de la columna posterior presente ${ }^{5,20,63}$. Existe adecuada evidencia también para el tratamiento incruento de Fracturas por Compresión y Lesiones por Flexión-Distracción Transóseas de un solo nivel $\left.\right|^{5,6,18,45,65,66}$.

Acorde con estos datos, nuestra conducta fue entonces tratar ortopédicamente las Fracturas por Compresión, las Estallido Estables (subtipos A, B y C de Denis) independientemente de la ocupación del canal, Lesiones por Flexión-Distracción Transóseas de un nivel (subtipo A de Denis), correspondientes con los Tipo A, B.2.1 y algunas B.2.3 de Magerl ${ }^{65}$. En el sacro tratamos así las fracturas sin déficit o con déficit menor estable y sin compresión actual, en las verticales ubicadas en las zonas I y || de Denis, y las transversas de la zona III bajas sin déficit ${ }^{67}$.

El agrupar las lesiones vertebrales de nuestra serie en traumas de Alta y Baja Energía define dos patrones significativamente diferentes de transformación de la energía cinética durante el impacto, en donde la masa del cuerpo y la velocidad del impacto son las variables más importantes de dichos patrones ${ }^{7}$. Los requerimientos ortésicos de ambos tipos de patrones son diferentes, especialmente 
cuando los trauma de baja energía ocurren en huesos insuficientes $^{50,68}$. En nuestra serie solos 6 de las 19 fracturas del Grupo B se presentaron en huesos aparentemente competentes, pero dado que: a) la presencia de insuficiencia ósea puede ser incluso enmascarada en traumas de alta energía ${ }^{29}$, b) los patrones morfológicos y por ende los requerimientos biomecánicos son similares, y que c) en el presente estudio no se realizó ninguna medición de la densidad mineral ósea, nos parece oportuna la diferenciación terapéutica en dichos grupos.

Nuestro tratamiento se basó en la utilización de corsets de yesos seguido ortesis rígidas (TLSO) hechas a medida en los traumas de alta energía, y ortesis en hiperextensión prefabricadas (Corset de Jewett) en los de baja energía. Ambas actúan parcialmente en el modo "load-sharing" pero fundamentalmente como sistemas de inmovilización pasivos de 3 o 4 puntos ${ }^{6,69}$ que cumplen su rol por restricción de los movimientos groseros del tronco durante la cicatrización ósea ${ }^{6,14,69,70}$. Su efectividad biomecánica depende por tanto de las fuerzas correctivas que ejercen ${ }^{6}$. Las ortesis en hiperextensión prefabricadas tipo Jewett no han demostrado, sin embargo, en otros trabajos adecuada efectividad en el control de los parámetros radiológicos en las fracturas estallido en hueso normal12,71. Comparativamente las TLSO termoplásticas hechas-a-medida, son las que proveen un mejor control de todos los movimientos ${ }^{6,11}$; tomamos sus moldes en Mesa para Yeso EDF tipo Cotrel para emplear mejor las fuerzas correctoras ${ }^{18,72}$.

La efectividad del manejo ortésico de las fracturas tóracolumbares puede ser evaluada con los resultados en los parámetros geométrico y funcionales: reducción del ángulo de cifosis postraumático, disminución del porcentaje de compresión vertical sagital, disminución del porcentaje del compromiso del canal, ausencia de cambios o mejoría del estado neurológico, nivel de dolor, mejoría o retorno a las actividades de la vida diaria, y retorno a las actividades laborales ${ }^{6}$.

La corrección radiológica inicial con métodos incruentos no se mantiene pero siempre exhibe una ligera mejoría respecto al valor inicial ${ }^{14}$. Es corriente ver una pérdida angular en la corrección incruenta de la cifosis pero sin deterioro clínico ${ }^{4,17,61,63,72}$. En nuestra serie los valores angulares a la presentación y al seguimiento no exhibieron diferencias significativas en ninguno de los subgrupos de pacientes; por tanto, no obtuvimos a largo plazo ni mejoría ni deterioro radiológico de las lesiones, y obviamente hubo diferencias significativas respecto a los valores del grupo control (ver Tablas 1 y 4). Se colige que aunque fundado en principios biomecánicos sólidos el tratamiento ortésico de estas fracturas, no logran un resultado radiológico mantenido en el tiempo de los parámetros angulares correspondientes a la cifosis y pérdida de altura corporal.

Al seguimiento la comparación del "ABC" del grupo control y de los subgrupos de fracturas según la energía del trauma mostraron siempre mayor diferencia estadística en los subgrupos de baja energía que en aquellos de alta energía (Tabla 4). Esto habla de un resultado más cercano a "lo normal" (Grupo C) para los pacientes con trauma de Alta Energía. Hipotéticamente, esto podría deberse a una acción más eficaz por parte del tipo tratamiento ortésico realizado en dicho grupo a diferencia del usado en el trauma de baja energía.

De todas formas, está demostrado que la severidad de la lesión radiográfica inicial o residual no se correlaciona con los síntomas al seguimiento ${ }^{73}$ y que la cifosis secuelar no se correlaciona bien con los resultados clínicos independientemente del tipo de tratamiento $4,5,17,27,36,63,66$. Cabe destacar, por otra parte, que los promedios de la cifosis al seguimiento en los dos subgrupos de nuestra serie quedaron bastante por debajo del valor de corte de $30^{\circ}$ dado por el informe de la SRS ${ }^{38}$.

Nuestro mismo grupo de trabajo reportó anteriormente ${ }^{13} 87 \%$ de Resultados Clínicos Buenos con un excelente intervalo de confianza (77.8\%-96.2\%), para el tratamiento conservador de las fracturas tóraco-lumbares. Ellos se comparan favorablemente con las series internacionales ${ }^{63,64,70,72}$. En la presente serie, se confirman los buenos resultados clínicos hallados entonces tanto para el valor promedio global del Score de la SRS para el dolor en el follow-up (4.4), como el promedio del Oswestry Disability Index (15) y el porcentaje de retorno a la actividad previa (90\%) (Ver Tabla 5 y Figura 4). Ahora bien, la comparación nos mostró que los pacientes del Grupo A tuvieron un mejor resultado clínico que aquellos con trauma de Baja Energía (Dolor según SRS p 0.005, Oswestry Disability Index p 0.0002). Posiblemente esta diferencia se deba a factores tales como la mayor edad promedio del Grupo B ( $p$ 0.0001) asociada a patologías subyacentes no analizadas en el presente trabajo. En ellos, al ser las características de los tejidos subyacentes diferentes y menores en calidad comparativamente a las del Grupo A, la cantidad de movimiento y la energía cinética asociadas con el impacto productor de la lesión son también inferiores ${ }^{7-10}$. Por otra parte, en dicho tipo de pacientes son reconocidas las ventajas de un reposo breve con movilización precoz y del uso de ortesis prefabricadas menos constreñidas mucho mejor toleradas y más rápidamente accesibles comparadas con las hechas a medida ${ }^{6,68,74}$. Por lo tanto, teniendo en cuenta estas consideraciones es lícito pensar que siendo el resultado funcional bueno en el Grupo B - a pesar de su menor calidad respecto al del Grupo A -, la selección de ortesis prefabricadas es costo-beneficio efectiva para los pacientes con trauma de baja energía cinética.

\section{CONCLUSIONES}

1) Es posible lograr un Resultado Clínico Funcional satisfactorio a mediano plazo en las lesiones tóraco-lumbosacras mayores estables sin lesión neurológica progresiva seleccionando el tipo de ortesis según que el trauma sea de alta o baja energía cinética.

2) Los resultados clínicos funcionales parecen ser mejores en los casos de Trauma de Alta Energía, aunque los mismos parecen estar asociados a factores independientes del tipo de ortesis.

3) Sin embargo, el tratamiento ortésico diferenciado según alta energía o baja energía no logra en el tiempo un resultado radiológico cercano a lo normal los parámetros geométricos.

\section{AGRADECIMIENTOS}

Los autores desean expresar su agradecimiento a los Dres. Alfredo Zurita (Prof. Titular de Metodología de la Investigación \& Prof. Titular de Salud Pública - Facultad de Medicina - UNNE - Corrientes) y Joan Bagó Granell (Barcelona - ESPAÑA), así como al Licenciado en Física Mario Cleva (U.T.N. Facultad Regional Resistencia - Resistencia - Chaco) por la ayuda y guía recibida tanto en la preparación y evaluación del manuscrito como en el procesamiento y revisión estadística del presente trabajo. 


\section{REFERENCIAS}

1. Rowley DI. Enhancement of the healing of Fractures. In: Thorngren KG, Soucacos PN, Horan, Scott J. European Instructional Course Lectures. London: The British Society of Bone and Joint Surgery; 2001. p. 24-30.

2. Dai LD. Low lumbar spinal fractures: management options. Injury. 2002;33(7):579-82.

3. Stadhouder A, Buskens E, de Klerk LW, Verhaar JA, Dhert WA, Verbout AJ, et al. Traumatic thoracic and lumbar spinal fractures: operative or nonoperative treatment: comparison of two treatment strategies by means of surgeon equipoise. Spine (Phila Pa 1976). 2008;33(9):1006-17.

4. Yi L, Jingping B, Gele J, Baoleri X, Taixiang W. Operative versus non-operative treatment for thoracolumbar burst fractures without neurological deficit. Cochrane Database Syst Rev. 2006;(4):CD005079.

5. Spivak JM, Vaccaro AR, Cotler JM. Thoracolumbar Spine Trauma: II. Principles of Management. J Am Acad Orthop Surg. 1995;3(6):353-360.

6. Malas BS, Meade KP, Patwardhan AG, Gavin TM. Orthoses for spinal trauma and postoperative care. In: Hsu JD, Michael JW, Fisk JR, editors. AAOS atlas of orthotic an assistive devices. 4th ed. Philadelphia: Mosby-Elsevier; 2008. p. 141-53.

7. Hunt JP, Weintraub SL, Wang YZ, Buechter KJ. Kinematics of trauma. In: Moore EE, Feliciano DV, Mattox KL, editors. Trauma. New York: McGraw-Hill Professional; 2003. p. 141-57.

8. Neumann P, Osvalder AL, Nordwall A, Lövsund P, Hansson T. The ultimate flexural strength of the lumbar spine and vertebral bone mineral content. J Spinal Disord. 1993;6(4):314-23.

9. Pintar FA, Yoganandan N, Voo L. Effect of age and loading rate on human cervical spine injury threshold. Spine (Phila Pa 1976). 1998;23(18):1957-62.

10. Tran NT, Watson NA, Tencer AF, Ching RP, Anderson PA. Mechanism of the burst fracture in the thoracolumbar spine. The effect of loading rate. Spine. 1995;20(18):1984-8.

11. Lantz SA, Schultz AB. Lumbar spine orthosis wearing. I. Restriction of gross body motions. Spine (Phila Pa 1976). 1986;11(8):834-7.

12. Bago J, Villanueva C, Aguirre M, Marti D. Tratamiento conservador de las fracturas estallidos vertebrales. Estudio comparativo entre el Corsé de yeso y la ortesis de Jewett. Rev Orthop Traumatol. 1990;34(6):605-7.

13. Manzone $P$, Mariño Ávalos $E$, Cardozo Iñiguez $L$, Quiroz J, Laluf A, et al.: Tratamiento no Quirúrgico de las Fracturas Toraco-Lumbosacras. In: XIV Congreso de la Sociedad Argentina de Patología de la Columna Vertebral; 2006, Mar del Plata - Argentina, 12, 13 y 14 de Octubre de 2006

14. Weninger $P$, Schultz $A$, Hertz H. Conservative management of thoracolumbar and lumbar spine compression and burst fractures: functional and radiographic outcomes in $136 \mathrm{ca}-$ ses treated by closed reduction and casting. Arch Orthop Trauma Surg. 2009;129(2):20719

15. Smith JA, Siegel JH, Siddiqi SQ. Spine and spinal cord injury in motor Vehicle crashes: a function of change in velocity and energy dissipation on impact with respect to the direction of crash. J Trauma. 2005:59(1):117-31.

16. Bazán PL, Borri A, Feldman D, Szmidt VE. Evaluación cinemática del trauma raquimedular. In: IX Congreso de la Sociedad Ibero latinoamericana de la Columna \& XXI Congreso Nacional de la Sociedad para el Estudio de las Enfermedades del Raquis (GEER); 2007; Santander - España, 31 de Mayo al 02 de Junio de 2007.

17. Alanay A, Yazici M, Acaroglu E, Turhan E, Cila A, Surat A. Course of nonsurgical management of burst fractures with intact posterior ligamentous complex: an MRI study. Spine (Phila Pa 1976). 2004;29(21):2425-31.

18. Chapman MW. Fracture healing and closed treatment of fractures and dislocations. In: Chapman's orthopaedic surgery. 3rd ed. Philadelphia: Lippincott Williams \& Wilkins; 2001. p. 219-305.

19. Cantor JB, Lebwohl NH, Garvey T, Eismont FJ. Nonoperative management of stable thoracolumbar burst fractures with early ambulation and bracing. Spine (Phila Pa 1976). 1993;18(8):971-6.

20. Chow GH, Nelson BJ, Gebhard JS, Brugman JL, Brown CW, Donaldson DH. Functiona outcome of thoracolumbar burst fractures managed with hyperextension casting or bracing and early mobilization. Spine (Phila Pa 1976). 1996;21(18):2170-5

21. Fiore $\mathrm{N}$, Iraporda H. Fracturas de columna lumbar. Tratamiento incruento. Presentación de 5 casos. Revista de la AAOT. 2001;66(2):128-30.

22. Shen WJ, Shen YS. Nonsurgical treatment of three-column thoracolumbar junction burst fractures without neurologic deficit. Spine (Phila Pa 1976). 1999;24(4):412-5.

23. Panjabi MM, Thibodeau LL, Crisco JJ 3rd, White AA 3rd. What constitutes spinal instability? Clin Neurosurg. 1988;34:313-39.
24. Farcy JP, Weidenbaum M, Glassman SD. Sagittal index in management of thoracolumbar burst fractures. Spine (Phila Pa 1976). 1990;15(9):958-65.

25. Lim M, Vaccaro A, Lee JY, Zeiller S, Jacoby S, SanFilippo J, et al. The thoracolumbar injury severity scale and score (TLISS): inter-physician and inter-disciplinary validation of a new paradigm for the treatment of thoracolumbar spine trauma. Coluna/Columna. 2006:5(3):157-64.

26. Denis F, Davis S, Comfort T. Sacral fractures: an important problem. Retrospective analysis of 236 cases. Clin Orthop Relat Res. 1988;227:67-81.

27. Domenicucci M, Preite R, Ramieri A, Ciappetta P, Delfini R, Romanini L. Thoracolumbar fractures without neurosurgical involvement: surgical or conservative treatment? J Neurosurg Sci. 1996;40(1):1-10

28. Magerl F, Aebi M, Gertzbein SD, Harms J, Nazarian S. A comprehensive classification of thoracic and lumbar injuries. Eur Spine J. 1994;3(4):184-201.

29. Sanders KM, Pasco JA, Ugoni AM, Nicholson GC, Seeman E, Martin TJ, Skoric B, Panahi $\mathrm{S}$, Kotowicz MA. The exclusion of high trauma fractures may underestimate the prevalence of bone fragility fractures in the community: the Geelong Osteoporosis Study. $J$ Bone Miner Res. 1998;13(8):1337-42.

30. Alanay A, Pekmezci M, Karaeminogullari O, Acaroglu E, Yazici M, Cil A, et al. Radiographic measurement of the sagittal plane deformity in patients with osteoporotic spinal fractures evaluation of intrinsic error. Eur Spine J. 2007;16(12):2126-32.

31. Enad JG, Slakey JB, McNulty PS. Measurement of thoracolumbar kyphosis after burst fracture: evaluation of intraobserver, interobserver, and variability of 4 measurement methods. Am J Orthop (Belle Mead NJ). 2008;37(4):E60-3.

32. Keynan O, Fisher CG, Vaccaro A, Fehlings MG, Oner FC, Dietz J, Kwon B, Rampersaud R, Bono C, France J, Dvorak M. Radiographic measurement parameters in thoracolumbar fractures: a systematic review and consensus statement of the spine trauma study group. Spine (Phila Pa 1976). 2006;31(5):E156-65.

33. Kuklo TR, Polly DW, Owens BD, Zeidman SM, Chang AS, Klemme WR. Measurement of thoracic and lumbar fracture kyphosis: evaluation of intraobserver, interobserver, and technique variability. Spine (Phila Pa 1976). 2001;26(1):61-5.

34. Goutallier D, Hernigou P, Piat C. [Orthopedic treatment of fractures of the dorsolumbar and lumbar spine with recoil of the posterior corpus wall (burst fracture) without or with minor neurologic lesions]. Rev Chir Orthop Reparatrice Appar Mot. 1988;74(Suppl 2):77-83.

35. Roy-Camille R, Saillant G, Massin P. [Treatment of fracture of the thoracolumbar spine using Böhler's method]. Rev Chir Orthop Reparatrice Appar Mot. 1989;75(7):479-89

36. Weinstein JN, Collalto P, Lehmann TR. Thoracolumbar "burst" fractures treated conservatively: a long-term follow-up. Spine (Phila Pa 1976). 1988;13(1):33-8.

37. Willén J, Lindahl S, Nordwall A. Unstable thoracolumbar fractures. A comparative clinical study of conservative treatment and Harrington instrumentation. Spine (Phila Pa 1976). 1985;10(2):111-22.

38. Gertzbein SD. Scoliosis Research Society. Multicenter spine fracture study. Spine (Phila Pa 1976). 1992;17(5):528-40.

39. Pueyrredon MA, Scharovsky AP, Craig D. Adaptación Cultural y Validación para la Argentina del Oswestry Disability Index. Poster presentado en el XII World Congreso on Pain, Glasgow, Scotland; 2008.

40. Livingston $\mathrm{EH}$. Who was student and why do we care so much about his t-test? J Surg Res. 2004;118(1):58-65.

41. Petrie A. Statistics in orthopaedic papers. J Bone Joint Surg Br. 2006;88(9):1121-36.

42. Szabo RM. Principles of epidemiology for the orthopaedic surgeon. J Bone Joint Surg Am. 1998:80(1):111-20

43. Coggon D, Rose G, Barker D. Epidemiología. 3a. ed. Chile: Publicaciones Técnicas Mediterráneo; 1993. p.107-10.

44. Aligizakis AC, Katonis PG, Sapkas G, Papagelopoulos PJ, Galanakis I, Hadjipavlou A. Gertzbein and load sharing classifications for unstable thoracolumbar fractures. Clin Orthop Relat Res. 2003;(411):77-85.

45. Mirza SK, Mirza AJ, Chapman JR, Anderson PA. Classifications of thoracic and lumbar fractures: rationale and supporting data. J Am Acad Orthop Surg. 2002;10(5):364-77.

46. Wood KB, Khanna G, Vaccaro AR, Arnold PM, Harris MB, Mehbod AA. Assessment of two thoracolumbar fracture classification systems as used by multiple surgeons. J Bone Joint Surg Am. 2005;87(7):1423-9.

47. Denis F. The three column spine and its significance in the classification of acute thoracolumbar spinal injuries. Spine (Phila Pa 1976). 1983;8(8):817-31.

48. Denis F. Spinal instability as defined by the three-column spine concept in acute spinal 
trauma. Clin Orthop Relat Res. 1984;(189):65-76.

49. McAfee PC, Yuan HA, Lasda NA. The unstable burst fracture. Spine (Phila Pa 1976). 1982:7(4):365-73.

50. McAfee PC, Yuan HA, Fredrickson BE, Lubicky JP. The value of computed tomography in thoracolumbar fractures. An analysis of one hundred consecutive cases and a new classification. J Bone Joint Surg Am. 1983;65(4):461-73.

51. McCormackT, Karaikovic E, Gaines RW. The load sharing classification of spine fractures. Spine (Phila Pa 1976). 1994;19(15):1741-4

52. Louis R. Les theories de l'instabilité. Rev Chir Orthop. 1977;63:415-81.

53. An HS, Simpson JM, Ebraheim NA, Jackson WT, Moore J, O'Malley NP. Low lumbar burst fractures: comparison between conservative and surgical treatments. Orthopedics. 1992;15(3):367-73

54. An HS, Vaccaro A, Cotler JM, Lin S. Low lumbar burst fractures. Comparison among body cast, Harrington rod, Luque rod, and Steffee plate. Spine (Phila Pa 1976). 1991;16(8 Suppl):S440-4.

55. Dai $L Y$, Jiang SD, Wang $X Y$, Jiang $L S$. A review of the management of thoracolumbar burst fractures. Surg Neurol. 2007;67(3):221-31.

56. Dai LY, Jiang LS, Jiang SD. Conservative treatment of thoracolumbar burst fractures: a long-term follow-up results with special reference to the load sharing classification. Spine (Phila Pa 1976). 2008;33(23):2536-44

57. Hitchon PW, Torner JC, Haddad SF, Follett KA. Management options in thoracolumbar burst fractures. Surg Neurol. 1998;49(6):619-26.

58. Kinoshita $H$, Nagata $Y$, Ueda $H$, Kishi $K$. Conservative treatment of burst fractures of the thoracolumbar and lumbar spine. Paraplegia. 1993;31(1):58-67.

59. Knight RQ, Stornelli DP, Chan DP, Devanny JR, Jackson KV. Comparison of operative versus nonoperative treatment of lumbar burst fractures. Clin Orthop Relat Res. 1993;(293):112-21.

60. Kraemer WJ, Schemitsch EH, Lever J, McBroom RJ, McKee MD, Waddell JP. Functional outcome of thoracolumbar burst fractures without neurological deficit. J Orthop Trauma. 1996;10(8):541-4.

61. Moller A, Hasserius R, Redlund-Johnell I, Ohlin A, Karlsson MK. Nonoperatively treated burst fractures of the thoracic and lumbar spine in adults: a 23- to 41-year follow-up. Spine J. 2007;7(6):701-7.

62. Seybold EA, Sweeney CA, Fredrickson BE, Warhold LG, Bernini PM. Functional outcome of low lumbar burst fractures. A multicenter review of operative and nonoperative treat- ment of L3-L5. Spine (Phila Pa 1976). 1999;24(20):2154-61.

63. Shen WJ, Liu TJ, Shen YS. Nonoperative treatment versus posterior fixation for thoracolumbar junction burst fractures without neurologic deficit. Spine (Phila Pa 1976). 2001;26(9):1038-45

64. Wood K, Buttermann G, Mehbod A, GarveyT, Jhanjee R, Sechriest V, Butterman G. Operative compared with nonoperative treatment of a thoracolumbar burst fracture without neurological deficit. A prospective, randomized study. J Bone Joint Surg Am. 2003;85A(5):773-81.

65. Gertzbein S. Management of thoracolumbar fractures. In: Curso Oficial de la Sociedad Argentina de Patología de la Columna Vertebral. Controversias en Cirugía Espinal; Argentina, 6 y 7 de junio 2003. p.18.

66. Hazel WA Jr, Jones RA, Morrey BF, Stauffer RN. Vertebral fractures without

neurological deficit. A long-term follow-up study. J Bone Joint Surg Am. 1988;70(9):1319-21.

67. Kim MY, Reidy DP, Nolan PC, Finkelstein JA. Transverse sacral fractures: case series and literature review. Can J Surg. 2001:44(5):359-63.

68. Meade KP, Malas BS, Patwardhan AG, Gavin TM. Orthoses for osteoporosis. In: Hsu JD, Michael JW, Fisk JR, editors. AAOS atlas of orthotic an assistive devices. 4th ed. Philadelphia: Mosby-Elsevier; 2008. p. 155-66.

69. Lonner B, Hoppenfeld S, Patel A. Thoracolumbar spine fractures. In: Hoppenfeld S, Murphy VL. Treatment and rehabilitation of fractures. Philadelphia: Lippincott-Williams \& Wilkins; 2000. p. 561-74

70. Krag MH, Fox MS J, Haugh LD. Comparison of three lumbar orthoses using motion assessment during task performance. Spine (Phila Pa 1976). 2003;28(20):2359-67.

71. Nagel DA, Koogle TA, Piziali RL, Perkash I. Stability of the upper lumbar spine following progressive disruptions and the application of individual internal and external fixation devices. J Bone Joint Surg Am. 1981;63(1):62-70.

72. Tropiano P, Huang RC, Louis CA, Poitout DG, Louis RP. Functional and radiographic outcome of thoracolumbar and lumbar burst fractures managed by closed orthopaedic reduction and casting. Spine (Phila Pa 1976). 2003;28(21):2459-65.

73. Mumford J, Weinstein JN, Spratt KF, Goel VK. Thoracolumbar burst fractures. The clinical efficacy and outcome of nonoperative management. Spine (Phila Pa 1976). 1993;18(8):955-70.

74. Vives M, Garfin SR. Spinal orthoses for traumatic and degenerative disease. In: In: Herkowitz HN, Garfin S, Eismont FJ, Bell GR, editors. Rothman-Simeone the spine. 5th ed. Philadelphia Saunders; 2006. p.1203-19. 